\title{
Research Paper \\ Predicting Job Satisfaction Based on Personality Traits and Psychological Empowerment in Employed Middle-Aged and Elderly People
}

\author{
${ }^{*}$ Peyman Hatamian ${ }^{1} \odot$, Mojtaba Ahmadi Farsani ${ }^{2} \odot$, Jahangir Karami ${ }^{3} \odot$, Parastoo Hatamian ${ }^{4}$
}

1. Department of Psychology, Faculty of Humanities, Tarbiat Modares University, Tehran, Iran.

2. Department of Psychology, Faculty of Educational \& Psychology, Tabriz University, Tabriz, Iran

3. Department of Psychology, Faculty of Social Sciences, Razi University, Kermanshah, Iran.

4. Department of Economy, Faculty of Social Sciences, Razi University, Kermanshah, Iran.

\begin{tabular}{|c|c|}
\hline $\begin{array}{l}\text { Use yourd device to scan } \\
\text { and read the article online }\end{array}$ & dtation Hatamian P, Ahmadi Farsani M, Karami J, Hatamian P. [Predicting Job Satisfaction Based on Personality Traits \\
\hline aripa & $\begin{array}{l}\text { and Psychological Empowerment in Employed Middle-Aged and Elderly People (Persian)]. Iranian Journal of Ageing. 2019; } \\
\text { 13(4):418-427. https://doi.org/10.32598/SIJA.13.4.418 }\end{array}$ \\
\hline 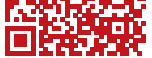 & d ci"https://doi.org/10.32598/SIJA.13.4.418 \\
\hline
\end{tabular}

(c) $0(3)$

Received: 16 Apr 2018

Accepted: 25 Oct 2018

Available Online: 01 Jan 2019

Keywords:

Personality Traits,

Empowerment, Job

Satisfaction, Elderly

\section{A B STRACT}

Objectives The associated factors with job satisfaction can predict the personal and professional progress of middle-aged and elderly people, as well as their future success. The present study aimed to predict job satisfaction based on personality traits and psychological empowerment among middle-aged and elderly working people.

Methods \& Materials Of all middle-aged and elderly employed people in Kermanshah City, Iran, a sample of 240 people were selected by the convenience sampling method. The study data were collected using NEO five-factor inventory, short form, psychological empowerment questionnaire and job satisfaction survey. For data analysis, the Pearson correlation coefficient and regression analysis methods were used. Results The results revealed that personality characteristics of extrovertism, accountability and flexibility were positively associated with job satisfaction with correlation coefficients of $0.42,0.34$, and 0.07 , respectively. In addition, there was a positive and significant relationship between psychological empowerment and job satisfaction with correlation coefficient of 0.43 . However, no significant relationship was found between mental health and pleasure with job satisfaction.

Conclusion According to the findings of this study, personality traits and psychological empowerment can predict job satisfaction in middle-aged and elderly people.

\section{Extended Abstract}

\section{Objectives}

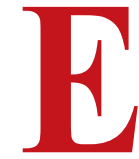

mployment is one of the issues that has always been one of the main concerns of people, states, and nations. In every country, there are thousands of jobs and professions that people are engaged in [1]. The people's job satisfaction and the effects of this satisfaction on their morale and productivity have always been considered by psychologists and social scientists [1]. The increasing growth of adult and elderly people in society and the employment of a large number of them, indicate the need for further research on their mental health. There is a paucity of research on the association between and Job Satisfaction (JS) and variables of Personality Traits (PTs) and Psychological Empowerment (PE), in employed middle-aged and elderly people. Thus, this study examined the rela-

\section{* Corresponding Author:}

Peyman Hatamian, MSc.

Address: Department of Psychology, Faculty of Humanities, Tarbiat Modares University, Tehran, Iran.

Tel: +98 (918) 9968103

E-mail: hatamian152@gmail.com 
tionship between these variables. We explored whether personality traits and psychological empowerment in employed middle-aged and elderly people can predict their job satisfaction.

\section{Methods and Materials}

This was a descriptive correlational study. Study population consisted of all middle-aged and elderly people employed in various occupational sectors (public and private) in Kermanshah City, Iran, in 2016. Of these, 240 samples were selected using convenience sampling method. To collect data, the following questionnaires were used: Job Satisfaction Survey (JSS) with 36 items; NEO Five-Factor Inventory (NEO-FFI), short form with 60 items developed by Costa and McCrae (1989). The Cronbach alpha coefficient for the reliability of the Persian version of NEO-FFI, short form have been reported as $0.76,0.65,0.59,0.48$, and 0.75 [2]; Psychological Empowerment Questionnaire (PEQ) with 20 items designed by Spreitzer (1995). He reported the Cronbach alpha coefficient of 0.85 for this tool.

After obtaining consent from the study participants and assuring them of the confidentiality of their information, the questionnaires were distribute among them. The collected data were analyzed by SPSS using Pearson correlation coefficient and regression analysis. This study was approved by the Department of Psychology of Razi University.

\section{Results}

The Mean \pm SD age of 240 samples was $66.89 \pm 1.93$ years. Almost $67 \%$ of the samples were male. The Kolmogorov-Smirnov test results suggested the normality of data distribution. To test the correlation between PTs and PE, and JS, Pearson correlation coefficient was used. The results revealed that, the correlation coeffi- cient for neuroticism and JS was $\mathrm{r}=-0.29$; for extraversion and JS, $r=0.42$; for openness and JS, $r=0.07$; for agreeableness and JS, $\mathrm{r}=0.38$, and for conscientiousness and JS, $r=0.43$. For evaluating the predictability of JS based on PTs and PE, simultaneous multiple regression analysis was used. According to the results, both PTs and PE can predict $18 \%$ of changes in JS. Among 5 PTs, conscientiousness, extraversion, and openness dimensions could predict JS with beta values of 0.18 , 0.11 , and 0.19 , respectively. PE with beta value of 0.37 was also able to predict JS. Therefore, among predicting variables, PE is more likely to predict JS (Table 1).

Overall, results demonstrate a significant positive relationship between extraversion and JS. This indicates that extroverts do not blame themselves for the mistakes. They do not get disappointed. They are active, hardworking and courageous, and take the right decisions in critical situations, and are more satisfied with their jobs. Results also indicate a significant positive relationship between JS and dimensions of conscientiousness and openness. However, neuroticism and agreeableness had no significant correlation with JS. PE also had a significant correlation with JS in the middle-aged and elderly people. Thus, JS increases with the increase in PS and vice versa.

\section{Conclusion}

Personality traits and psychological empowerment in the middle-aged and elderly people had a significant relationship with their job satisfaction. This is consistent with the findings of Karuk et al. [4], Birneh et al. [5], Caves and Demur [6], and other similar studies [7-10]. According to the results of the current study, personality traits (conscientiousness, extraversion, and openness) and psychological empowerment can predict job satisfaction in employed middle-aged and elderly people. Therefore, we suggest that managers and employers

Table 1. Regression results for evaluating the predictability of JS

\begin{tabular}{ccccc}
\hline Variable & Model Summary & Predictor & B & Sig. \\
\hline $\mathrm{R}=0.47$ & Conscientiousness & 0.23 & 0.18 \\
$\mathrm{JS}$ & Adjusted $\mathrm{R}^{2}=0.18$ & Extraversion & 0.01 & 0.11 \\
$\mathrm{~F}=31.17$ & Openness & 0.001 & 0.19 \\
$\mathrm{P}=0.001$ & $\mathrm{PE}$ & 0.32 & 0.04 & 0.37
\end{tabular}


consider the impact of these factors on job satisfaction of their staff. The samples had different personality traits. Thus, their degree of collaboration in research also varied depending on their personal and working conditions. This point was a limitation to our study.

\section{Ethical Considerations}

\section{Compliance with ethical guidelines}

In this paper, ethical guidelines including awareness of participants about the research process, confidentiality of their information, and the right to withdraw the study at any time were observed. The consent obtained from them was verbal.

\section{Funding}

Authors received no financial support.

\section{Authors contributions}

Conceptualization, formal analysis, validation, and draft preparation: Peyman Hatamian; Investigation and methodology: Parastoo Hatamian; Editing: All authors; Resources and funding acquisition: Mojtaba Ahmadi Farsani; and Supervision: Jahangir Karami.

\section{Conflict of interest}

The authors declared no conflict of interest. 


\title{
ييشبينى رضايت شغلى بر اساس ويزَّى هاى شخصيتى و توانمندسازى روانشناختى بين

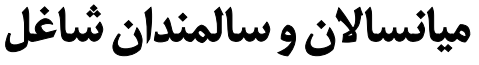

\author{
• بيمان حاتميان' •، مجتبى احمدى فارسانى' •، جهانكير كرمى' • يرستو حاتميان \\ ا - كروه روانشناسى، دانشكده علوم انسانى، دانشكاه تربيت مدرس، تهران، ايران.

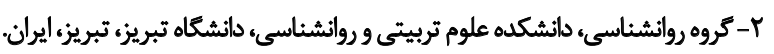

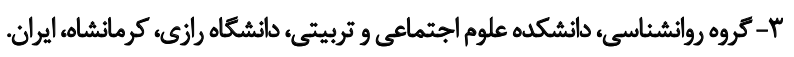 \\ †- كروه اقتصاد، دانشكده علوم اجتماعى و تربيتى، دانشكاه رازى، كرمانشاه، ايران.
}

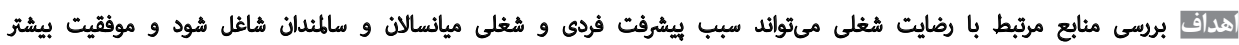

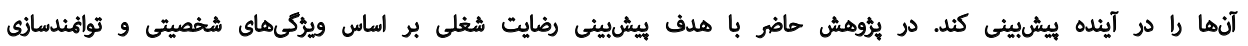

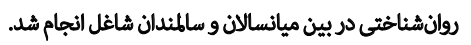

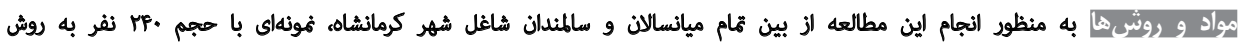

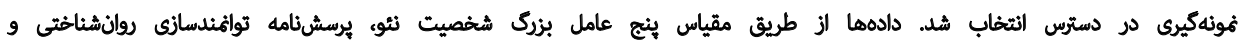

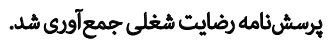

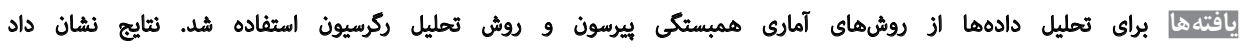

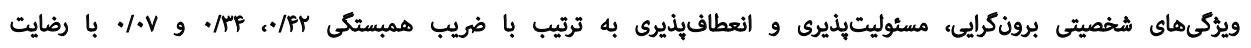

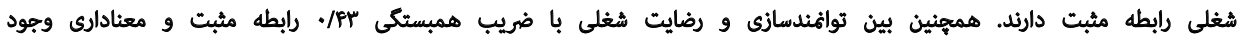

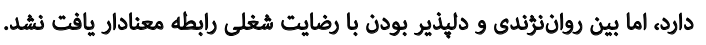

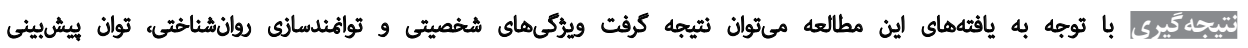

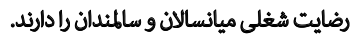

حكب

يكى از مهمترين موضوعاتى كه در هر سازمان بايد به آن توجه

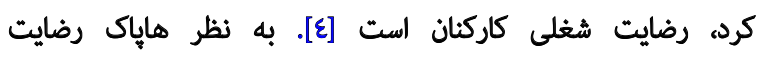

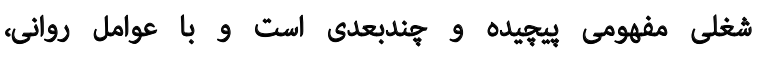

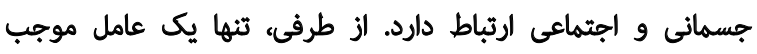

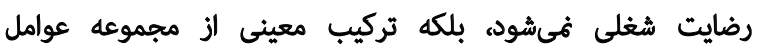

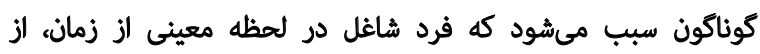
شغل اعساس رضايت كند و لذت بيرد [0].

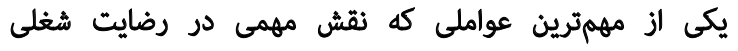

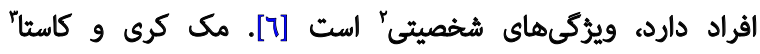

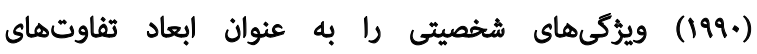

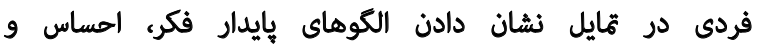

2. Personality traits

3. McCrae \& Costa

$$
\text { …… }
$$

اشتغال از جمله مسائلى است كه همواره ذهن انسانها، دولتها

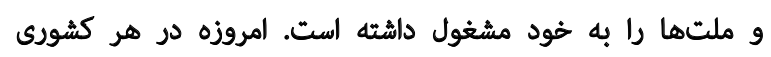

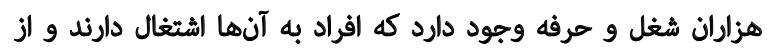

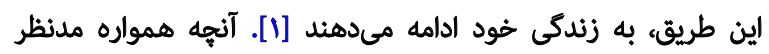

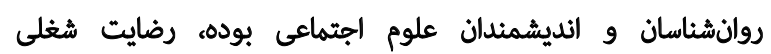

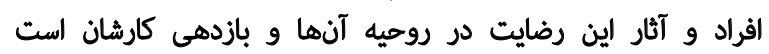

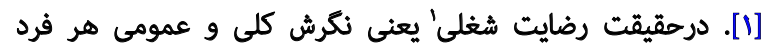

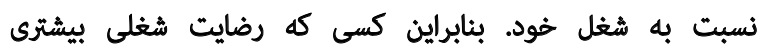

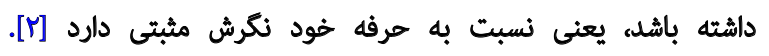

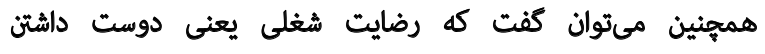
شرايط و لوازم يك شغل؛ شرايطى كه در آن كار انجام و ياداشى كانى

1. Job satisfaction

$$
\begin{aligned}
& \text { " يويسئده مسئول: } \\
& \text { بيمان حاتميان } \\
& \text { نشائى: تهران، دانشكاه تربيت مدرس، دانشكده علوم انسانى، كروه روانشناسى. }
\end{aligned}
$$

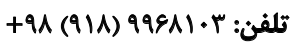

$$
\begin{aligned}
& \text { بست إلكترونيكى: hatamian152@gmail.com }
\end{aligned}
$$


كستردى عظيم قشر ميانسال و سالمند در جامعه و نيز شاغل

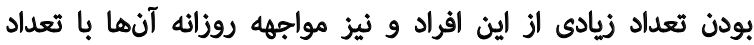

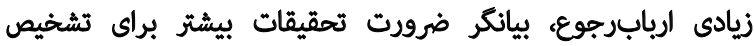

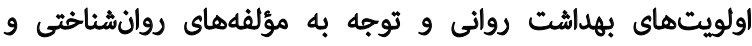

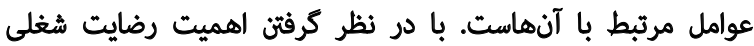

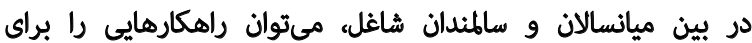
افزايش جنبههاى مثبت توانمندسازى روانشناختى ارائه كرد. اكرجها در يُروهشهاى كذشته ئروهشكران بر نقش تواتمثلدسازى

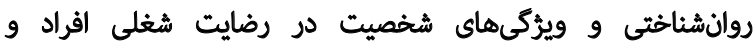

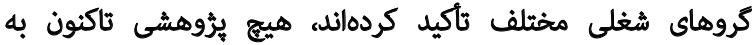

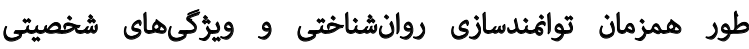

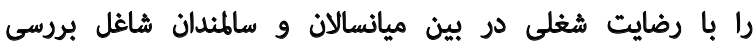

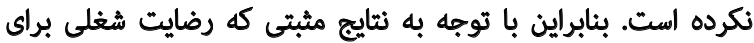

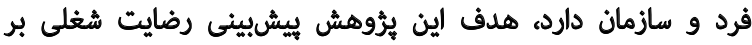

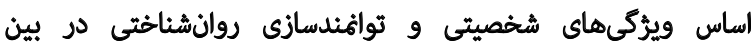
ميانسالان و سالمندان شاغل شهر كرمائشاه بود.

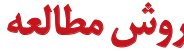

يثزوهش حاضر مطالعهاي توصيفى همبستكى بود. جامعه

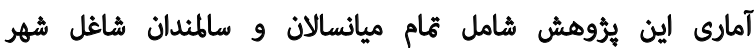

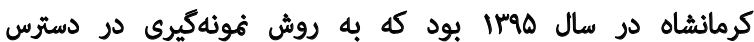

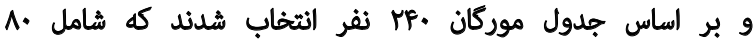

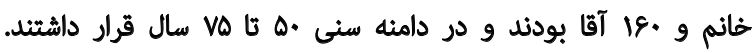

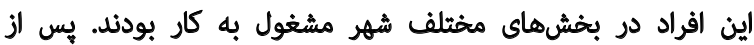

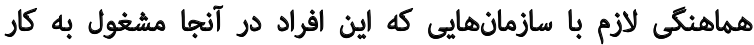

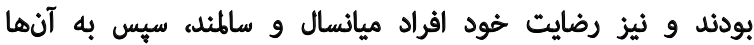

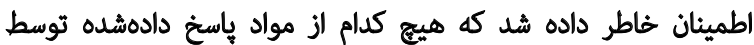

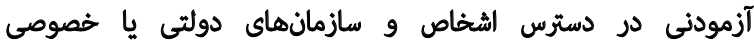

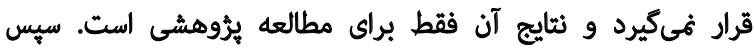

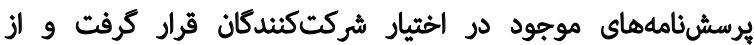

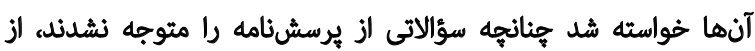

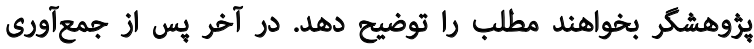

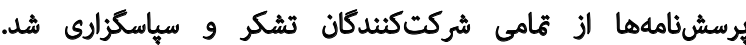

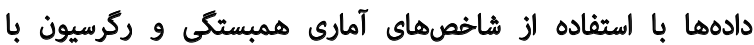

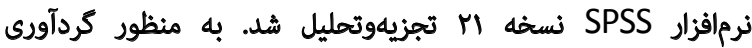
دادههاى يُّوهش از ابزارهاى زير استفاده شد.

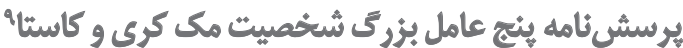

در اين يزوهش از فرم كوثاه آزمون نثو مككرى و كاستا

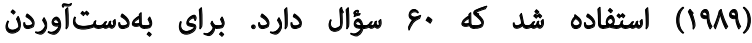

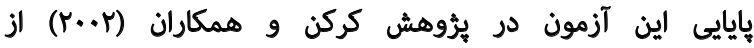

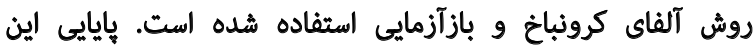

9. McCrae and Costa personality big five personality inventory (NEO)
عمل تعريف مىكند [V]

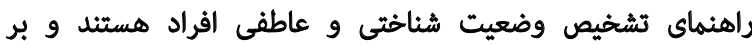

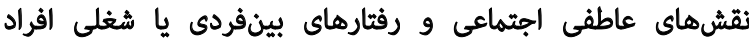

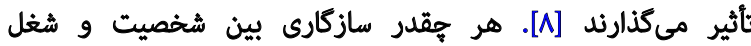

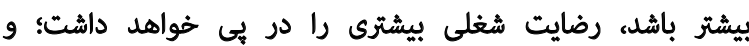

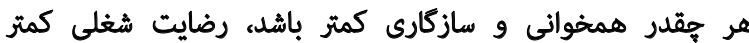

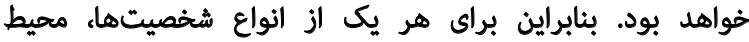

شغلى متفاوتى مناسب است [9].

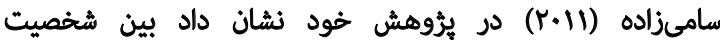

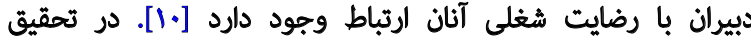

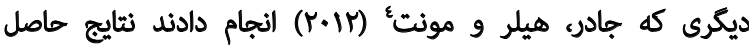

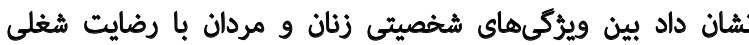

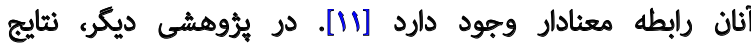

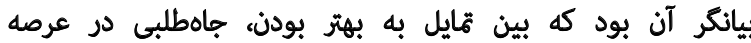

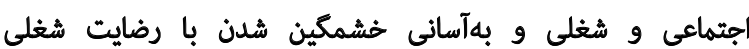

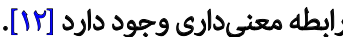

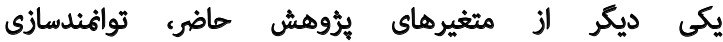

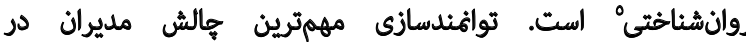

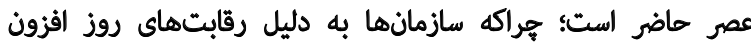

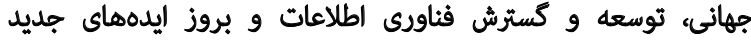

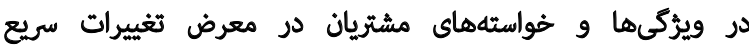

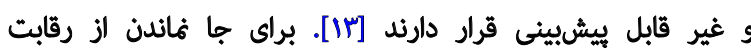

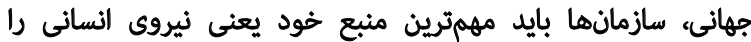

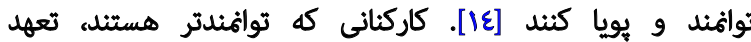

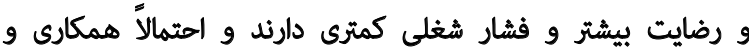

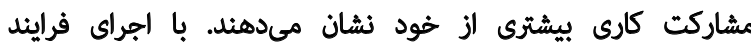

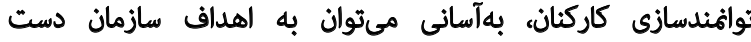

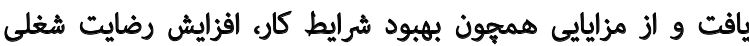

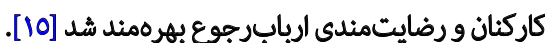

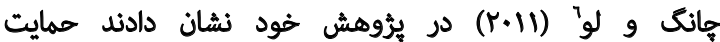

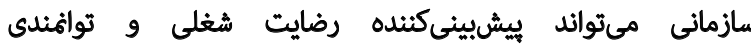

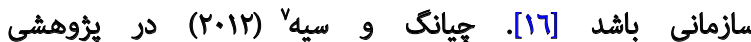

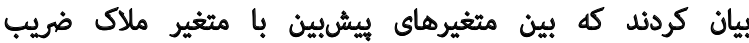

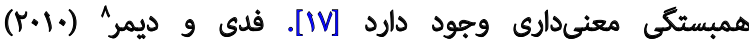

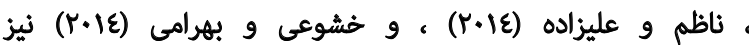
در يروهشهاى خود به اين نتيجه رسيدند كه بين توانمئدسازى روانشناختى و رضايت شغلى رابطه مثبت و و بين تواتمندسازى باني

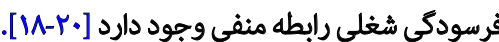

4. Judge, Heller \& Mount

5. Psychological empowerment

6. Chang \& Liu

7. Chiang \& Hsieh

8. Fade \& Demur 
مثبت جملهائ است كه موافقت با آن نشاندهنده رضايت از شغل

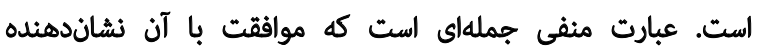

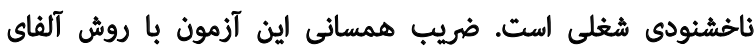

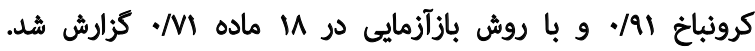

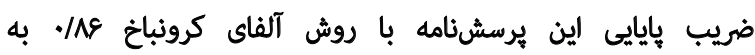
دست آمد [ست]

Ldiọt

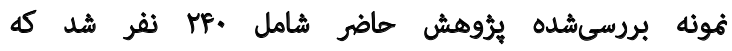

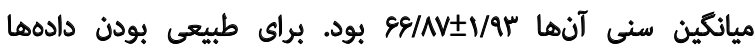

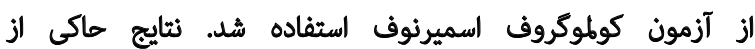
طبيعى بودن دادهها بود. در اين نهونه تقريباً

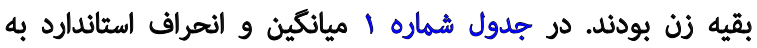
تفكيك كروه آمده الست.

براى بررسى رابطه بين ويرّكىهاى شخصيتى و توانمندى روانى

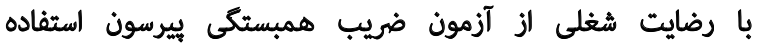

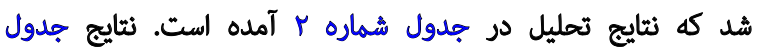

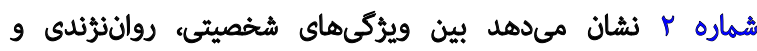

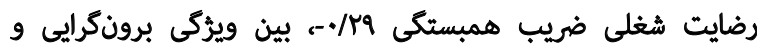

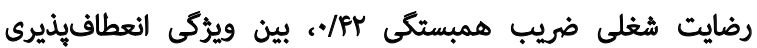

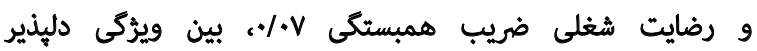

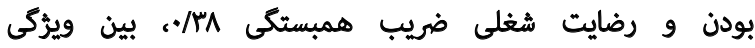

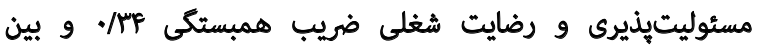

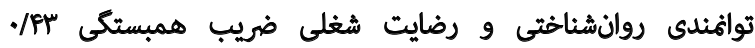

وجود دارد.

براى بيسشبينى رضايت شغلى بر الساس ويزكىهاي شغصيتى

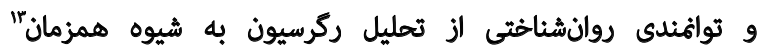

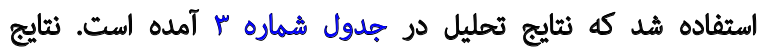

13. Enter
آزمون با استفاده از روش بازآزمايى روى Sf نفر از افراد نهونه

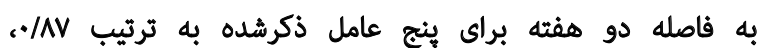
عاءAF عامل به ترتيب NG/.

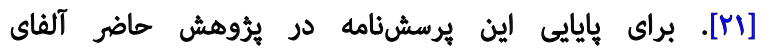

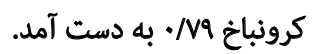

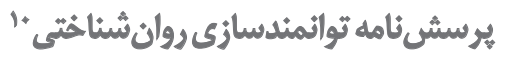

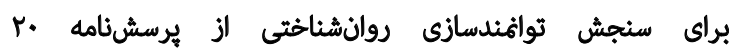

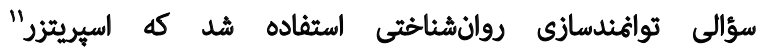

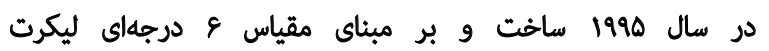

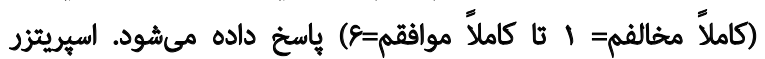

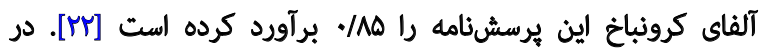

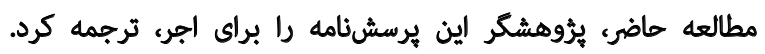

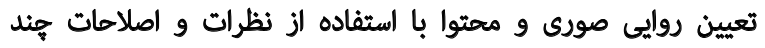

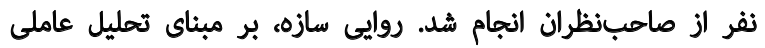

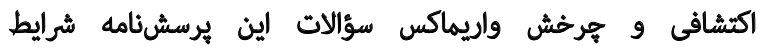

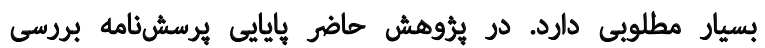

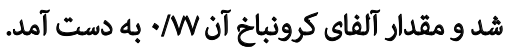

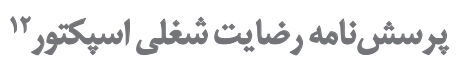

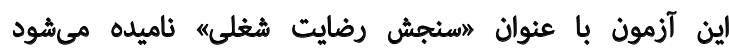

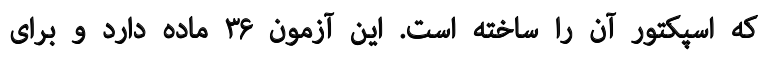

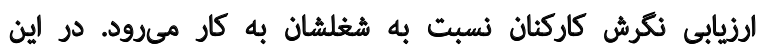

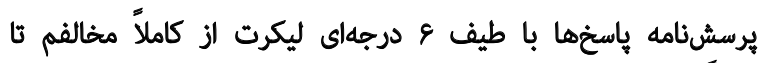

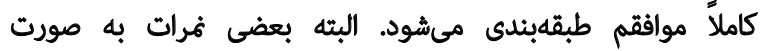

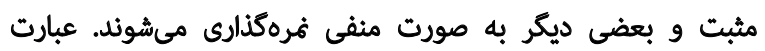

10. Psychological Empowerment Questionnaire 11. Spreitzer

12. Job Satisfaction Survey (JSS)

جدول ا. يافتههاى توصيفى براي متغيرهاى يروهش در نمونه بررسىشده

\begin{tabular}{|c|c|c|}
\hline اتحراف استاندارد & مياتكين & متغير \\
\hline$P / 11$ & $11 / r r$ & روانترثنلى \\
\hline$r / .9$ & $\mid \varepsilon / \pi$ & برونكرايى \\
\hline$\varphi / \Delta$ & WrA & انعطافيذيرى \\
\hline r/AT & $18 / 4$ & دليذير بودن \\
\hline$\Delta / r \Delta$ & me/me & مسئوليتيذيرى \\
\hline$r / 19$ & $r \cdot / l f$ & توانمثلدسازى روانشناختى \\
\hline$r / \Lambda$. & $19 / 99$ & رضايت شغلى \\
\hline
\end{tabular}


جدول r. ضرايب همبستكى بين ويرَكى هاى شخصيتى و توانمندى روانشناختى با رضايت شغلى

\begin{tabular}{|c|c|c|}
\hline سطح معنادارى & ضريب همبستكى & متغير \\
\hline.$/ \circ V$ & $-\infty / r q$ & روان رنثندى \\
\hline $.1 .+1$ &.$/ F r$ & برون كرايى \\
\hline $.1 .0 \%$ & $.1 \cdot v$ & انعطافيذيرى \\
\hline $.1 \cdot n$ &.$/ r A$ & دليذير بودن \\
\hline .1 .01 &.$/ m f$ & هسئوليتيذيرى \\
\hline $.1 . .1$ &.$/ p H$ & توائمندسازى روان شثاختي \\
\hline
\end{tabular}

24

جدول ץ. نتايج تحليل ركرسيون براى بيشبينى رضايت شغلى

\begin{tabular}{|c|c|c|c|c|c|}
\hline معنادارى & $\beta$ & B & متغير بيشبين & خلاصه مدل & مثغير ملكى \\
\hline .1 .1 &.$/ M$ &.$/ \pi$ & مستوليتيذيرى & $\mathrm{R}=* / \mathrm{eV}$ & \multirow{4}{*}{ رضايت شغلى } \\
\hline $.1+.1$ &.$/ 11$ &.$/ M f$ & برون كرايى & $\mathrm{R}^{2}=\star / \mathrm{A}$ & \\
\hline$\cdot 1 \cdot \varphi^{e}$ & .119 & 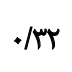 & انعطاف يذيرى & $F=r / / I V$ & \\
\hline $.1+.1$ &.$/ \pi V$ & $\cdot / \Delta F$ & توانمندسازى روان شُناختى & $\mathrm{P}=. / . .1$ & \\
\hline
\end{tabular}

24

رابطه مثبت و معنىدارى وجود دارد، ولى بين رواننزئنى و دليذير بودن با رضايت شغلى رابطه معنادار يافت نشد.

نثايج بهدستآمده الز يُوهش حاضر با نثايج يزوهشهاى سامىزاده (Y.1.) (Y.11)

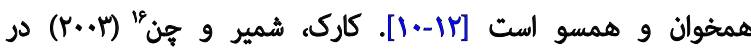

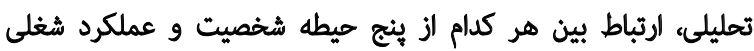

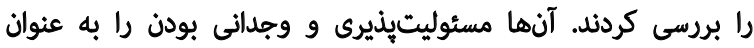

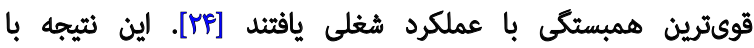

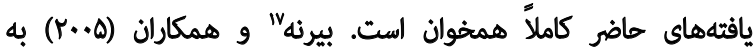

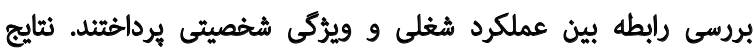

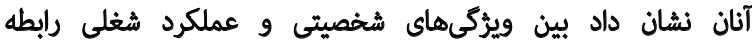

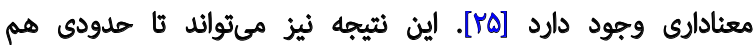

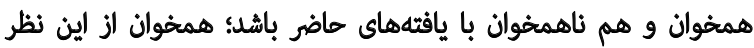

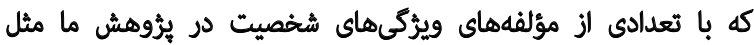

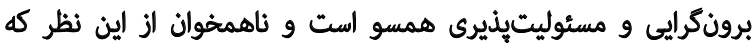

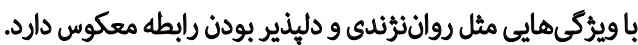

همهنين نتايج اين يُوهش نشان داد بين توائمندسازى و

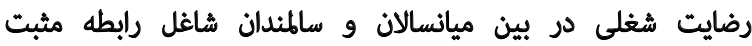

14. Judge

15. Ayah \& Kocacik

16. Karuk, Shamir \& Chen

17. Byrne
جدول شهاوه r نشان ميدهد ويزٔكىهاى شخصيتى و توانمندى

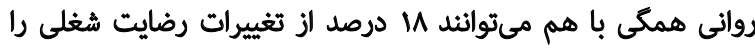

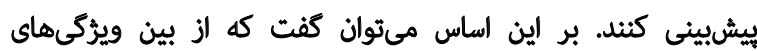

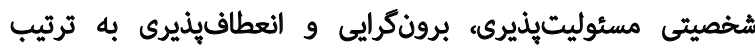

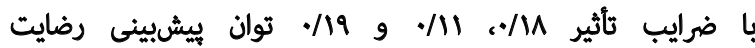

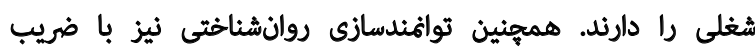

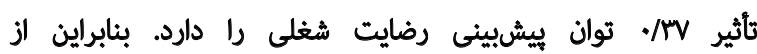

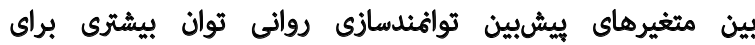
يويشبينى رضايت شغلى دارد.

\section{$\stackrel{4}{4}$}

يُروهش حاضر با هدف ييشبينى رضايت شغلى بر اساس

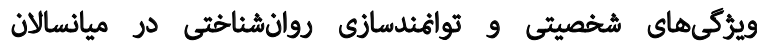

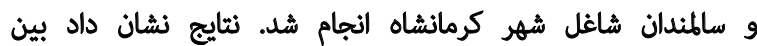

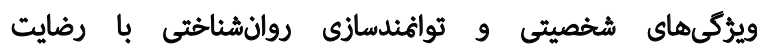

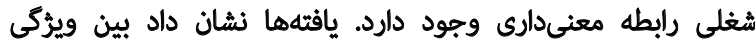

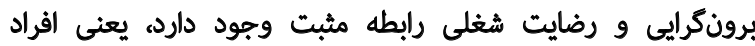

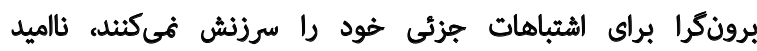

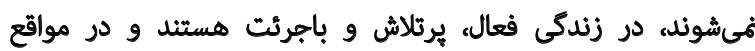

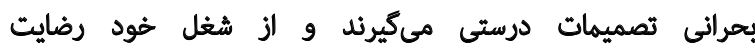

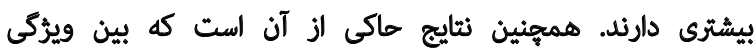

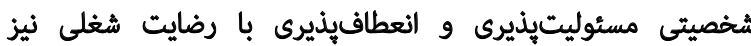


ويخٔكىهاي شخصيتى مثبت مثل الحساس مسنوليتيذيرى و هم

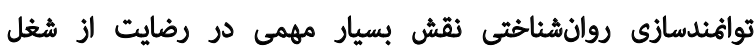

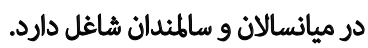

در زمينه محدوديتهاى ئزوهش بايد كفت كه هون غمونه

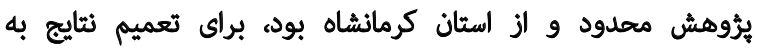

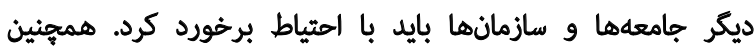

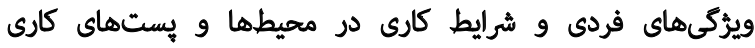

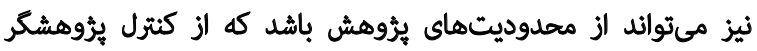
خارج بود. با توجه به الينكه يُووهش حاضر در بين ميانسالان و سالمندان

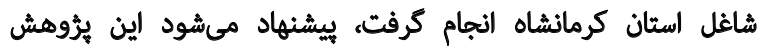
در استانهاى ديكر نيز النجام كيرد كه كار تعميم نثايج آساندان

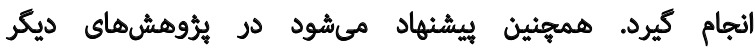

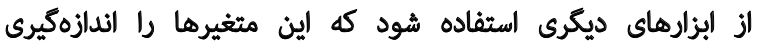

مىكند.

\section{ماحظات اخلاقى

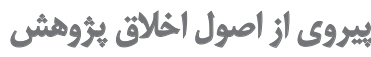

دريزوهش حاضر اصول اخلاقى مورد نياز در بزُوهش از جمله

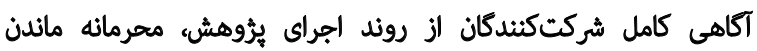

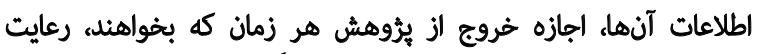

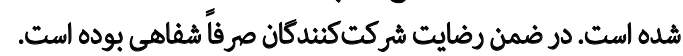

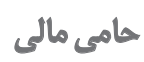

تمام هزينه هاى مريوط به بزؤهش حاضر توسط نويسندكان

$$
\text { تامين شده است. }
$$

$$
\text { مشاركت نويسند مان }
$$

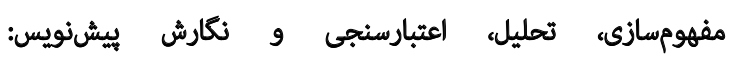

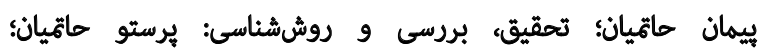

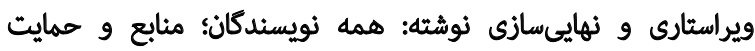

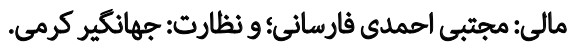

$$
\text { ت تعارض مناف }
$$

بنابر اظهار نويسندكان، اين مقاله تعارض منافع ندارد.

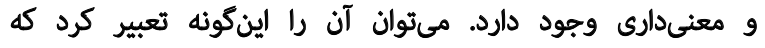

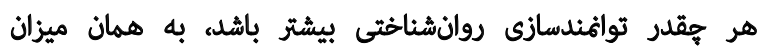

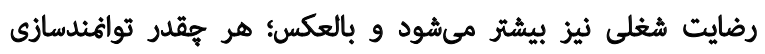

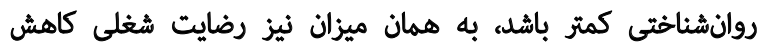

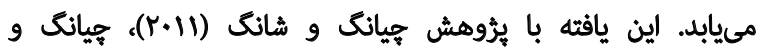

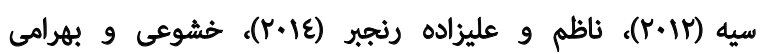

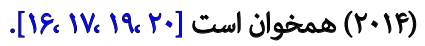

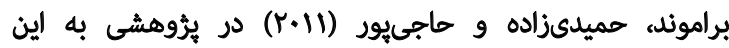

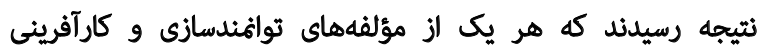

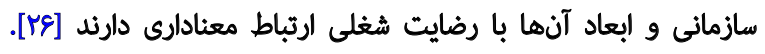

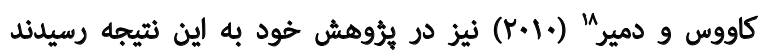

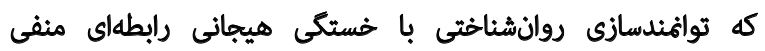

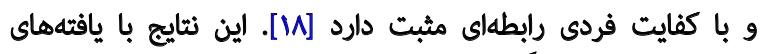

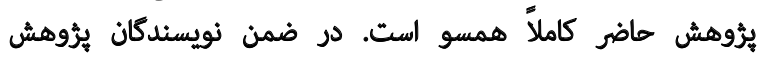

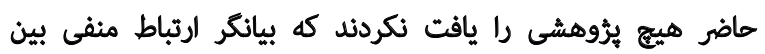

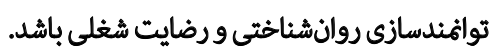

احساس تواثمندى، بحث نسبثاً جديدى است كه ثأثير فراوانى

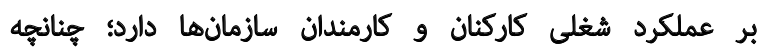

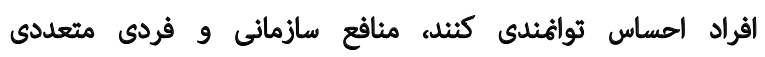

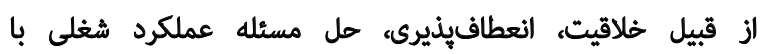

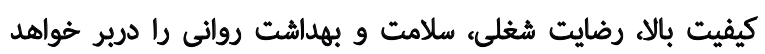

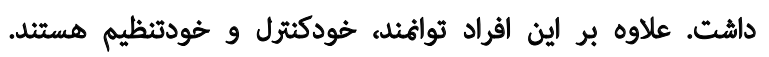

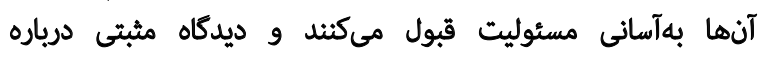

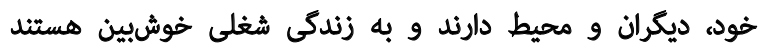

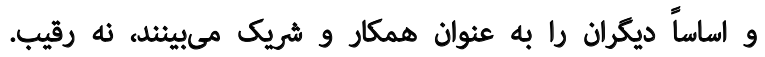

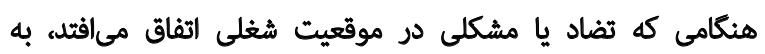

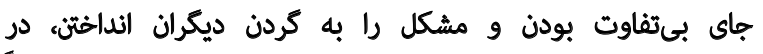

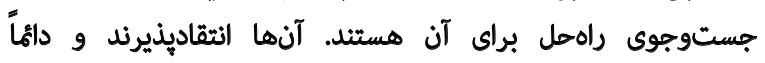
در حال يادكيرى هستند. مطالعات كسترده از از مثون تهقيقى

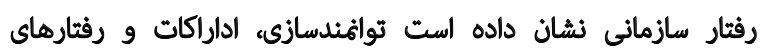

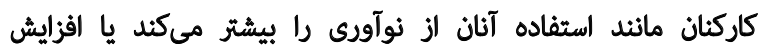

مي مهد [Mr]

$$
\text { نتيجهَيرى نهايى }
$$

نتايج حاصل از اين يزوهش و ئوهشهاى ديكر درباره رضايت

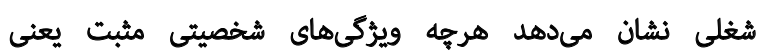

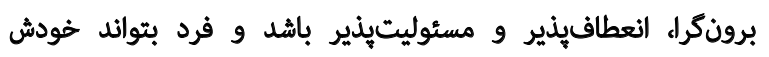

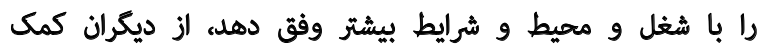

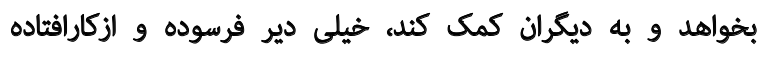

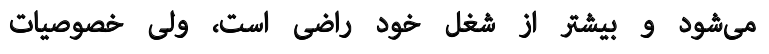

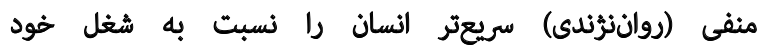

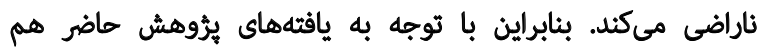

18. Caves \& Demur 


\section{References}

[1] George JM, Jones GR, Sharbrough WC. Understanding and managing organisational behavior. Boston, Massachusetts: Addison-Wesley; 2008.

[2] Robbins SP, Judge T. Essentials of organisational behavior. London: Pearson; 2005.

[3] Schertz BE. Career exploration and planning [M. Zandi Pour, Persian trans.]. Tehran: Ferdowsi.

[4] Luthans F, Youssef CM. Emerging positive organisational behavior. Journal of Management. 2007; 33(3):321-49.

[5] Kooshki Sh, Homan HA, Zahedi S. [The relationship between personality characteristics and job satisfaction in teachers (Persian)]. Psychological Research. 2009; 1(4):8-26.

[6] Moshabeky A, Rohani MH. [Management of organisational behavior (Persian)]. Tehran: Eghbal; 2009.

[7] Siyasi AA . [Ideas about personality (Persian)]. Tehran: Tehran University Press; 2012.

[8] Najarpor Ostadi S, Esmkhany Akbarinejad H, Lyvarjany Sh. [Studying the relations between personality characteristics \& irrational beliefs and job satisfaction among personnel of East Azerbaijan telecommunication department (Persian)]. Journal of Productivity Management. 2010; 4(13):39-58.

[9] Mostafaei F, Roshan R. [The relationship between personality features and job satisfaction of Tehran traffic police officers (Persian)]. Danesh-e Entezami. 2010; 11(4):183-206.

[10] Samizadeh A. [The relationship between personality type and personal characteristics and job satisfaction in middle school (Persian)] [MSc. thesis]. Kermanshah: Kermanshah University of Medical Sciences; 2011.

[11] Judge TA, Heller D, Mount MK. Five-factor model of personality and job satisfaction: A meta-analysis. Journal of Applied Psychology. 2002; 87(3):530-41. [PMID]

[12] Ayan S, Kocacik F. The relation between the level of job satisfaction and types of personality in high school teachers. Australian Journal of Teacher Education. 2010; 35(1):4. [DOI: 10.14221/ ajte.2010v35n1.4]

[13] Roy YJ, Sheena S. Empowerment through choice? A critical analysis of the effects of choice in organizations. Research in Organisational Behaviors. 2005; 27:41-79. [DOI: 10.1016/S01913085(06)27002-3]

[14] Ergeneli A, Arı GS, Metin S. Psychological empowerment and its relationship to trust in immediate managers. Journal of Business Research. 2007; 60(1):41-9. [DOI:10.1016/j.jbusres.2006.09.012]

[15] Shakerinia I, Nabavi SS. [Relationship between job satisfaction and perceived organisational support with organisational commitment among traffic and roads' police staff in Guilan Province (police job satisfaction) (Persian)]. Journal of Police Management Studies. 2011; 5(4):608-26.

[16] Chang LC, Liu CH. Employee empowerment, innovative behavior and job productivity of public health nurses: A crosssectional questionnaire survey. International Journal of Nursing Studies. 2008; 45(10):1442-8. [DOI:10.1016/j.ijnurstu.2007.12.006] [PMID]
[17] Chiang CF, Hsieh TS. The impacts of perceived organisational support and psychological empowerment on job performance: The mediating effects of organisational citizenship behavior. International Journal of Hospitality Management. 2012; 31(1):18090. [DOI:10.1016/j.j.jhm.2011.04.011]

[18] ÇAVUŞ MF, Demir Y. The impacts of structural and psychological empowerment on burnout: A research on staff nurses in Turkish state hospitals. Canadian Social Science. 2010; 6(4):63-72.

[19] Nazem F, Alizadeh Ranjbar T. [A study of the relationship of empowerment and working life quality to working exhaustion of personnel in education organization (Persian)]. Quarterly Journal of New Approaches in Educational Administration. 2014; 5(2):24-36.

[20] Khoshouei M, Bahrami M. [The role of psychological empowerment and organisational commitment in teachers' job burnout (Persian)]. Journal of School Psychology. 2014;3(1):37-54.

[21] Garosi MT, Ghazi Tabatabaei M, Mehryar AH. [Aplication of the NeoP1-R test and analytic evaluation of it's characteristics and factorial structure among Iranian University Students (Persian)]. Alzahra University Quarterly Journal of Humanities. 2001; 11(39):173-98

[22] Spreitzer GM. Psychological empowerment in the workplace: Demetrious, measurement and validation. Academy of Management Journal. 1995; 38(5):1442-65. [DOI:10.5465/256865]

[23] Van Saane N, Sluiter JK, Verbeek JH, Frings Dresen MH. Reliability and validity of instruments measuring job satisfaction: A systematic review. Occupational Medicine. 2003; 53(3):191-200. [DOI:10.1093/occmed/kqg038]

[24] Kark R, Shamir B, Chen G. The two faces of transformational leadership: Empowerment and dependency. Journal of Applied Psychology. 2003; 88(2):246-55. [PMID]

[25] Na'ami AZ. [Study of leadership styles and job satisfaction in employees of khuzestan steel mill (Persian)] [PhD. dissertation]. Ahwaz: Shahid Chamran University; 2005.

[26] Baramvand S, Hamidi Zadeh MR, Haji Pour B. [Investigate the relationship between entrepreneurship through empowerment and job satisfaction (Persian)]. Paper presented at: The National Conference on Entrepreneurship, Cooperation, Economic Jihad. 19 December 2011; Nain, Iran. 
This Page Intentionally Left Blank 